\title{
Abbau von UF-Harzen beim TMP- und CTMP-Aufschluss von mitteldichten Faserplatten (MDF)
}

\author{
Edmone Roffael · Brigitte Dix · Claus Behn · Gert Bär
}

Published online: 6 November 2008

(C) Die Autoren 2008. Dieser Artikel wurde mit Open Access auf Springerlink.com veröffentlicht.

\section{Degradation of UF-resins by thermo-mechanical and chemo-thermo-mechanical pulping of medium density fibreboards}

Subject Thermo-mechanical (TMP) and chemo-thermomechanical pulping (CTMP) of UF-bonded MDF induce considerable degradation of the UF-resin in the board and lead to a conspicuous increase in the content of water extractives of the fibres. Moreover, the water extractives of the fibres have a higher $\mathrm{pH}$-value and a lower buffering capacity as well as higher acetate and formate ions content than the extractives of the original board. However, the lignin content seems rather to increase than to decrease. This is possibly due to reaction between lignin and formaldehyde from the resin.

E. Roffael (汭)

Georg-August-Universität Göttingen,

Büsgenweg 4,

37077 Göttingen, Deutschland

E-Mail: eroffae1@gwdg.de

B. Dix

Wilhelm-Klauditz-Institut (WKI),

Fraunhofer-Institut für Holzforschung,

Bienroder Weg $54 \mathrm{E}$,

38108 Braunschweig, Deutschland

e-mail: brigitte.dix@wki.fraunhofer.de

C. Behn

Büsgen-Institut, Abteilung Molekulare Holzbiotechnologie und Technische Mykologie, Georg-August-Universität Göttingen,

Büsgenweg 2,

37077 Göttingen, Deutschland

G. Bär

Institut für Zellstoff und Papier (PTS-IZP),

Papiertechnische Stiftung (PTS),

Pirnaer Str. 37,

01809 Heidenau, Deutschland

\section{Material und Methodik}

UF-Harz-gebundene mitteldichte Faserplatten (MDF) mit einem Bindemittelanteil von 11,4\% (Feststoff/atro Fasern) wurden zum einen bei $170^{\circ} \mathrm{C}$ für 5 min thermo-hydrolytisch behandelt und anschließend in einem Einscheibenrefiner zerfasert. Zum anderen wurden die MDF bei $170^{\circ} \mathrm{C}$ für 5 min mit einem Gemisch aus 0,25\% Natriumhydroxid und $1 \%$ Natriumsulfit (jeweils bezogen auf atro Fasern) aufgeschlossen und ebenfalls in einem Einscheibenrefiner zerfasert. An den UF-Harz-gebundenen MDF sowie den hergestellten TMP- und CTMP wurde der Bindemittel-, Lignin- und Pentosangehalt bestimmt. Ferner wurde an den Kaltwasserextrakten der Fasern der Extraktstoffgehalt, der pH-Wert und die alkalische Pufferkapazität ermittelt (Tabelle 1).

Tabelle 1 Chemische Eigenschaften von UF-Harz-gebundenen MDF sowie deren TMP und CTMP

Table 1 Chemical properties of UF-bonded MDF and TMP and CTMP prepared therefrom

\begin{tabular}{|c|c|c|c|}
\hline \multirow[b]{2}{*}{ Eigenschaft } & \multirow[b]{2}{*}{ MDF } & \multicolumn{2}{|c|}{$\begin{array}{l}\text { Faserstoffe aus UF-Harz- } \\
\text { gebundenen MDF }\end{array}$} \\
\hline & & TMP & $\begin{array}{c}\text { CTMP } \\
(0,25 \% \mathrm{NaOH} \\
\left.1 \% \mathrm{Na}_{2} \mathrm{SO}_{3}\right)\end{array}$ \\
\hline Bindemittelanteil (\%) & 11,4 & 5,9 & 5,2 \\
\hline Ligningehalt (\%) & 27,4 & 28,1 & 30,4 \\
\hline Pentosangehalt (\%) & 7,4 & 6,6 & 5,9 \\
\hline Kaltwasserextraktgehalt (\%) & 6,9 & 10,4 & 11,2 \\
\hline pH-Wert des Kaltwasserextrakts & 4,8 & 6,3 & 6,4 \\
\hline $\begin{array}{l}\text { alkalische Pufferkapazität des } \\
\text { Kaltwasserextrakts } \\
\text { (mMol NaOH/100 g atro Faser) }\end{array}$ & 1,6 & 1,1 & 0,8 \\
\hline
\end{tabular}




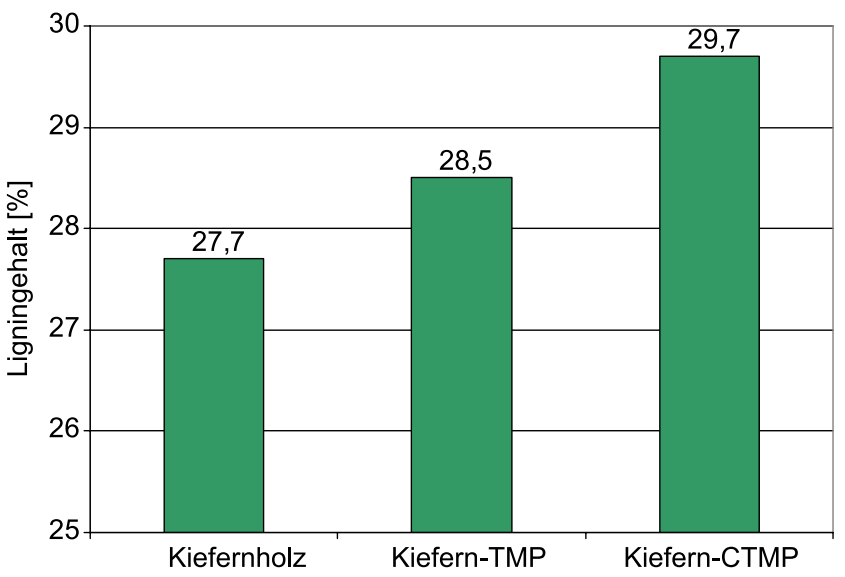

Abb. 1 Ligningehalt von Kiefernholz sowie von daraus hergestellten TMP- und CTMP

Fig. 1 Lignin content of pine wood as well as from TMP and CTMP prepared therefrom

\section{Ergebnisse und Diskussion}

Der Bindemittelanteil in den MDF lag bei $11,4 \%$ und fiel nach dem TMP-Aufschluss um 48,2\% auf 5,9\% ab. Weitaus stärker ist die Abnahme des Bindemittelgehalts durch den CTMP-Aufschluss, hier sank der Bindemittelanteil um 54,4\%. Der Abbau des UF-Harzes wird infolge der Bildung von alkalisch reagierenden Abbauprodukten des Harzes von einer Erhöhung des pH-Werts der wasserlöslichen Extrakte und einer Verringerung ihrer alkalischen Pufferkapazität begleitet (Franke und Roffael 1998). Interessant ist die Feststellung, dass auch unter diesen drastischen Aufschlussbedingungen keine vollständige Hydrolyse (Entfernung) des Bindemittels in den Platten möglich war. Darüber hinaus ist festzustellen, dass der Ligningehalt durch den Aufschluss eher erhöht als erniedrigt wurde. Eine gewisse Zunahme des Ligningehalts durch den Aufschluss bei hohen Temperaturen wurde auch im Falle von bindemittelfreiem Kiefernholz beobachtet (Abb. 1). Inwieweit dies allein mit dem Abbau der Hemicellulosen (s. Pentosangehalt) durch den Aufschluss in Zusammenhang steht und/oder ob eine Vernetzung des Lignins mit dem Formaldehyd (Sastry 1969) eine Rolle spielt, muss späteren Untersuchungen vorbehalten bleiben.

Danksagung Förderung: Das Verbundvorhaben zwischen der Universität Göttingen, dem Fraunhofer-Institut für Holzforschung und dem Institut für Zellstoff und Papier der Papiertechnischen Stiftung wurde mit Mitteln des Bundesministeriums für Wirtschaft und Technologie (BMWi) durch die Arbeitsgemeinschaft industrieller Forschungsvereinigungen (AiF- Nr. 14801 BG) über den Internationalen Verein für Technische Holzfragen (iVTH) gefördert.

Open Access Dieser Artikel wird zu den Bedingungen der "Creative Commons Attribution Noncommercial License" zur Verfügung gestellt. Damit ist eine nichtkommerzielle Nutzung, Verbreitung und Vervielfältigung erlaubt, sofern die Autoren des Artikels und die genaue Quelle angegeben sind.

\section{Literatur}

Franke R, Roffael E (1998) Zum Recycling von Span- und MDFPlatten. Teil 2: Einfluss der thermischen Behandlung von zerkleinerten UF-gebundenen Span- und MDF-Platten sowie Kiefernspänen auf die Formaldehyd- und Ammoniakabgabe. Holz Roh- Werkst 56:381-385

Sastry GP (1969) The reaction of formaldehyde with spruce lignin. Holzforschung 23:15-17 